\title{
Enhancing stimulated Raman excitation and two-photon absorption using entangled states of light
}

\author{
Anatoly Svidzinsky $\odot,{ }^{1}$ Girish Agarwal, ${ }^{1}$ Anton Classen, ${ }^{1}$ Alexei V. Sokolov $\odot,{ }^{1,2}$ \\ Aleksei Zheltikov, ${ }^{1,3,4}$ M. Suhail Zubairy, ${ }^{1}$ and Marlan O. Scully ${ }^{1,2}$ \\ ${ }^{1}$ Texas A\&M University, College Station, Texas 77843, USA \\ ${ }^{2}$ Baylor University, Waco, Texas 76798, USA \\ ${ }^{3}$ Moscow State University, Moscow 119992, Russia \\ ${ }^{4}$ Russian Quantum Center, Skolkovo 143025, Russia
}

(Received 18 December 2020; accepted 28 September 2021; published 12 October 2021)

\begin{abstract}
We find that stimulated Raman excitation of an atom by a two-photon pulse can be enhanced by orders of magnitude if the photons are simultaneously frequency correlated and spatially anticorrelated. That is, a correlated photon pair must have an inherent time delay between its constituent photons. This counterintuitive feature is a manifestation of the uncertainty principle, which yields that frequency-correlated photons cannot be time (spatially) correlated. This is opposite to two-photon absorption by a three-level atom, for which the enhancement occurs if photons in the pulse are frequency anticorrelated and spatially correlated, that is, photons in the pair simultaneously interact with the atom. Our findings can be useful for imaging and spectroscopy of biological samples which demand low illumination intensity.
\end{abstract}

DOI: 10.1103/PhysRevResearch.3.043029

Interaction of nonclassical radiation fields with atoms is a subject of long-standing interest. Entangled two-photon states of light have potential applications in quantum information processing [1], communications, and optical switching [2]. The resolution of images can be enhanced using two-photon absorption in conjunction with quantum imaging techniques $[3,4]$. Potential applications would benefit from new methods of enhancing the rate of two-photon processes. For example, two-photon absorption laser-induced fluorescence diagnostics is a powerful technique which has been used to provide information on densities of plasma and flame species [5]. It has been applied to atmospheric-pressure plasma jets to measure atomic oxygen and nitrogen densities [6-8], and to obtain temporally and spatially resolved hydrogen and oxygen images in nonequilibrium, nanosecond-duration pulsed-discharge plasmas [9].

The use of nonclassical states of light can open the way to realize a low-intensity microscopy at intensity levels not achievable with classical sources and to achieve the same fluorescence signal at much lower excitation light intensity [10]. This reduction of the probing light intensity is a critically important advantage for sensing [11] and biological applications since high light intensity could result in damage of sensitive chemical and biological samples.

For atomic systems, it has been shown that efficiencies of the two-photon absorption [12-18] and upconversion of light $[19,20]$ can be enhanced by using quantum correlated photon pairs. This effect has been observed experimentally

Published by the American Physical Society under the terms of the Creative Commons Attribution 4.0 International license. Further distribution of this work must maintain attribution to the author(s) and the published article's title, journal citation, and DOI.
[14,21,22]. Entangled photons can also induce transitions in two-atom systems [23,24] or induce two-photon transparency [25]. Recently, it has been demonstrated that arbitrary steadystate population inversion can occur in atoms driven by a squeezed vacuum reservoir [26]. Squeezed light can also enhance two-photon absorption [27] (see also Appendix).

The most widely used method for the generation of pairs of entangled photons is spontaneous parametric frequency downconversion, in which an incident pump photon effectively splits up in a nonlinear medium into two lower-frequency (signal and idler) photons. Parametrically down-converted light exhibits correlations between the down-converted photons, in that detection of a photon at one point in space-time may enormously enhance the probability of detecting its counterpart at another space-time location. At low photon fluxes, these correlations correspond to production of entangled photon pairs [28]. Two-photon excitation induced by entangled photons can exhibit nonclassical features, in particular, a linear dependence on the intensity $[12,13,29,30]$, which was observed with two-photon absorption [14,31].

Here, we consider a three-level atom with transition frequencies $\omega_{1}$ and $\omega_{2}$ between states $|c\rangle,|b\rangle$, and $|a\rangle$ (see Fig. 1) placed in a pulse of entangled light. Initially, the atom is in the ground state $|c\rangle$. We assume that the atom is being excited by a two-photon pulse described by the field state vector

$$
|i\rangle=\sum_{\mathbf{k}_{1}, \mathbf{k}_{2}} \phi\left(\mathbf{k}_{1}, \mathbf{k}_{2}\right)\left|1_{\mathbf{k}_{1}} 1_{\mathbf{k}_{2}}\right\rangle,
$$

where $\phi\left(\mathbf{k}_{1}, \mathbf{k}_{2}\right)$ is a probability amplitude to find two photons in the pulse with wave vectors $\mathbf{k}_{1}$ and $\mathbf{k}_{2}$. For the ladder $\Xi$ scheme of Fig. 1(a), the atom can become excited to the upper state $|a\rangle$ by a two-photon absorption. While for the Raman $\Lambda$ scheme of Fig. 1(b), the atom absorbs a photon from the pulse and then emits a photon of different frequency (Raman 
(a)

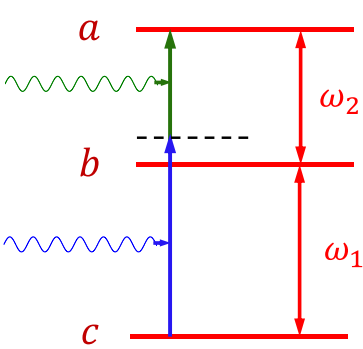

(b)

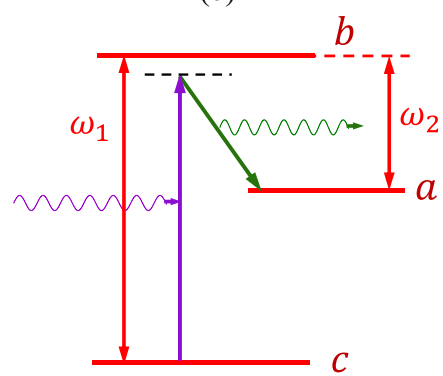

FIG. 1. A three-level atom with transition frequencies $\omega_{1}$ and $\omega_{2}$ between states $|c\rangle,|b\rangle$, and $|a\rangle$. The state $|c\rangle$ is coupled to $|b\rangle$, and $|b\rangle$ is coupled to $|a\rangle$. In the ladder scheme (a) the ground-state atom can become excited to the state $|a\rangle$ by absorbing two photons, while in the $\Lambda$ scheme (b) a photon is absorbed and reemitted with different frequency (Raman scattering).

scattering). In the Raman scattering the photon emission can be stimulated by the photon remaining in the pulse. We assume that both photons in the pulse have a relatively wide bandwidth, but the sum (or difference) of their frequencies is still well defined, such that the two-photon process is on resonance with the states $|c\rangle$ and $|a\rangle$. Here, we will consider a two-photon process via a virtual state without real excitation of the intermediate state $|b\rangle$, that is, photons in the pulse are detuned from the $c-b$ transition frequency.

The probability amplitude that the atom goes from the ground state $|c\rangle$ to the state $|a\rangle$ is given by

$$
A=-\frac{1}{\hbar^{2}} \sum_{f}\left\langle f\left|\left\langle a\left|\int_{-\infty}^{\infty} \hat{V}\left(t^{\prime}\right) d t^{\prime} \int_{-\infty}^{t^{\prime}} \hat{V}\left(t^{\prime \prime}\right) d t^{\prime \prime}\right| c\right\rangle\right| i\right\rangle,
$$

where $|i\rangle$ and $|f\rangle$ stand for the initial and final states of the field, respectively. In the rotating wave approximation the interaction Hamiltonian between the atom at $\mathbf{r}=0$ and the field for the ladder scheme reads

$$
\begin{aligned}
\hat{V}(t)= & -\wp_{b c} \sum_{\mathbf{k}} g_{k} \hat{a}_{\mathbf{k}} e^{i\left(\omega_{1}-v_{k}\right) t}|b\rangle\langle c| \\
& -\wp_{a b} \sum_{\mathbf{q}} g_{q} \hat{a}_{\mathbf{q}} e^{i\left(\omega_{2}-v_{q}\right) t}|a\rangle\langle b|+\text { H.c. },
\end{aligned}
$$

where $v_{k}=c k$ is the photon angular frequency, $\hat{a}_{\mathbf{k}}$ and $\hat{a}_{\mathbf{k}}^{\dagger}$ are annihilation and creation operators for the photon with wave vector $\mathbf{k}, \wp_{b c}$ and $\wp_{a b}$ are dipole moment matrix elements between the states $|b\rangle$ and $|c\rangle$, and $|a\rangle$ and $|b\rangle$, respectively, $g_{k}$ is the atom-photon coupling constant $g_{k}=\sqrt{\hbar v_{k} / 2 L_{\mathrm{ph}}^{3} \varepsilon_{0}}$, and $L_{\mathrm{ph}}$ is the photon length. In Eq. (2) the summation is over all possible final states of the field $f$. For the $\Lambda$ scheme, the second term in Eq. (3) should be replaced with

$$
-\wp_{a b} \sum_{\mathbf{q}} g_{q} \hat{a}_{\mathbf{q}}^{\dagger} e^{-i\left(\omega_{2}-v_{q}\right) t}|a\rangle\langle b| .
$$

Substituting Eq. (3) into Eq. (2) and integrating over time yields

$$
A_{ \pm}=\frac{2 \pi i \wp b c \wp a b}{\hbar^{2}} \sum_{\mathbf{k}, \mathbf{q}} \frac{g_{k} g_{q}}{\omega_{1}-v_{k}} \delta\left[\omega_{1}-v_{k} \pm\left(\omega_{2}-v_{q}\right)\right] M_{ \pm}
$$

where the upper sign and the lower sign in Eq. (4) correspond to the ladder $\Xi$ and the Raman $\Lambda$ scheme of Fig. 1, respectively. The $\delta$ function in Eq. (4) implies energy conservation in the two-photon process, and

$$
M_{+}=\sum_{f}\left\langle f\left|\hat{a}_{\mathbf{q}} \hat{a}_{\mathbf{k}}\right| i\right\rangle, \quad M_{-}=\sum_{f}\left\langle f\left|\hat{a}_{\mathbf{q}}^{\dagger} \hat{a}_{\mathbf{k}}\right| i\right\rangle .
$$

For the ladder scheme, the final state of the field is the state with zero photons, and using Eq. (1), we obtain

$$
M_{+}=\phi(\mathbf{q}, \mathbf{k})+\phi(\mathbf{k}, \mathbf{q}) .
$$

For the case of the $\Lambda$ scheme we will consider the stimulated Raman scattering process. Then, for $M_{-}$in Eq. (5), the final state of the field is the state with two photons in the mode $\mathbf{q}$, yielding $M_{-}=\sqrt{2} M_{+}$.

We consider a one-dimensional geometry in which the two-photon pulse propagates along the $x$ axis. This is a good approximation for collinear propagation of photons. Replacing the sum in Eq. (4) with an integral, we obtain

$$
\begin{aligned}
A_{ \pm}= & \frac{i c \wp_{b c} \bigodot_{a b}}{4 \pi \hbar \varepsilon_{0} L_{\mathrm{ph}}} \\
& \times \int_{-\infty}^{\infty} d k \int_{-\infty}^{\infty} d q \frac{\sqrt{|k q|}}{\left(\omega_{1}-v_{k}\right)} \delta\left[\omega_{1}-v_{k} \pm\left(\omega_{2}-v_{q}\right)\right] M_{ \pm} .
\end{aligned}
$$

We assume that $\phi(q, k)$ peaks at $k$ close to $\omega_{1} / c$ and $q$ close to $\omega_{2} / c$. Due to the factor $1 /\left(\omega_{1}-v_{k}\right)$, the first term in $M_{ \pm}$gives the main contribution to the integral in Eq. (7). Integration over $q$ yields

$$
A_{ \pm}=c L_{\mathrm{ph}} G_{ \pm} \int_{-\infty}^{\infty} d k \frac{\sqrt{\left|k q_{ \pm}\right|}}{\left(\omega_{1}-v_{k}\right)} \theta\left[\omega_{2} \pm\left(\omega_{1}-v_{k}\right)\right] \phi\left(q_{ \pm}, k\right)
$$

where $G_{+}=i \wp_{b c} \wp_{a b} / 4 \pi c \hbar \varepsilon_{0} L_{\mathrm{ph}}^{2}$ and $G_{-}=\sqrt{2} G_{+}$are dimensionless constants and $c q_{ \pm}=\omega_{2} \pm\left(\omega_{1}-v_{k}\right)$.

Next we discuss an upper limit on the atom's excitation probability by a separable two-photon state

$$
\phi\left(k_{1}, k_{2}\right)=f_{1}\left(k_{1}\right) f_{2}\left(k_{2}\right),
$$

where $f_{1}(k)$ and $f_{2}(k)$ are functions that peak at $k=k_{\alpha}$ and $k=k_{\beta}$, respectively. We assume that $k_{\alpha}$ and $k_{\beta}$ obey the condition of two-photon resonance

$$
k_{\beta} \pm k_{\alpha}=\frac{\omega_{1} \pm \omega_{2}}{c}
$$

and $c k_{\beta}\left(c k_{\alpha}\right)$ is close to $\omega_{1}\left(\omega_{2}\right)$, namely, $\left|c k_{\beta, \alpha}-\omega_{1,2}\right| \ll$ $\omega_{1,2}$. In terms of $k_{\alpha}$ and $k_{\beta}$ one can write $q_{ \pm}$as

$$
q_{ \pm}=k_{\alpha} \pm k_{\beta} \mp k,
$$

that is, if $k=k_{\beta}$, then $q_{ \pm}=k_{\alpha}$. The state (1) is normalized such that

$$
\int_{-\infty}^{\infty} \int_{-\infty}^{\infty} d k_{1} d k_{2}\left|\phi\left(k_{1}, k_{2}\right)\right|^{2}=\frac{(2 \pi)^{2}}{L_{\mathrm{ph}}^{2}}
$$

Plugging Eq. (9) into Eq. (8) yields

$$
A_{ \pm}=c L_{\mathrm{ph}} G_{ \pm} \int_{-\infty}^{\infty} d k \frac{\sqrt{\left|k q_{ \pm}\right|}}{\left(\omega_{1}-v_{k}\right)} \theta\left[\omega_{2} \pm\left(\omega_{1}-v_{k}\right)\right] f_{1}\left(q_{ \pm}\right) f_{2}(k) \text {. }
$$


The function $f_{1}\left(q_{ \pm}\right) f_{2}(k)$ peaks at $k=k_{\beta}$. Taking the factor in front of this function at $k=k_{\beta}$, the integral in Eq. (12) can be approximated as

$$
A_{ \pm} \approx c L_{\mathrm{ph}} G_{ \pm} \frac{\sqrt{k_{\alpha} k_{\beta}}}{\omega_{1}-c k_{\beta}} \int_{-\infty}^{\infty} d k f_{1}\left(k_{\alpha} \pm k_{\beta} \mp k\right) f_{2}(k) .
$$

Using the Cauchy-Schwarz inequality, one can write

$$
\begin{aligned}
& \left|\int_{-\infty}^{\infty} d k f_{1}\left(k_{\alpha} \pm k_{\beta} \mp k\right) f_{2}(k)\right| \\
& \quad \leqslant \sqrt{\int_{-\infty}^{\infty} d k\left|f_{1}\left(k_{\alpha} \pm k_{\beta} \mp k\right)\right|^{2}} \sqrt{\int_{-\infty}^{\infty} d k\left|f_{2}(k)\right|^{2}} \\
& \quad=\sqrt{\int_{-\infty}^{\infty} d k\left|f_{1}(k)\right|^{2}} \sqrt{\int_{-\infty}^{\infty} d k\left|f_{2}(k)\right|^{2}}=\frac{2 \pi}{L_{\mathrm{ph}}},
\end{aligned}
$$

where we used the normalization condition (11). As a result, we find the following upper bound on the excitation probability amplitude produced by the separable two-photon state:

$$
\left|A_{ \pm}\right| \leqslant 2 \pi c G_{ \pm} \frac{\sqrt{k_{\alpha} k_{\beta}}}{\omega_{1}-c k_{\beta}} .
$$

This upper bound can be achieved for a certain class of separable states.

Next we assume that the two-photon pulse is entangled and has the form

$$
\phi=\frac{N}{\left(k_{1}+k_{2}-k_{\alpha}-k_{\beta}\right)^{2}+\gamma_{+}^{2}} \frac{e^{i \Delta_{1} k_{1}+i \Delta_{2} k_{2}}}{\left(k_{1}-k_{2}-k_{\alpha}+k_{\beta}\right)^{2}+\gamma_{-}^{2}},
$$

where $N$ is a normalization constant, $k_{\alpha}$ and $k_{\beta}$ obey the condition of two-photon resonance (10), $c k_{\beta}\left(c k_{\alpha}\right)$ is close to $\omega_{1}\left(\omega_{2}\right), \gamma_{ \pm} \ll k_{\alpha, \beta}$, and $\Delta_{1,2}$ are parameters determining correlation between photons.

The normalization constant $N$ is obtained from Eq. (11), which yields $N=4 \sqrt{2}\left(\gamma_{+} \gamma_{-}\right)^{3 / 2} / L_{\text {ph. }}$. Plugging Eq. (15) into Eq. (8) gives

$$
\begin{aligned}
A_{ \pm}= & \frac{c L_{\mathrm{ph}} N G_{ \pm}}{\gamma_{ \pm}^{2}} e^{i \Delta_{1}\left(k_{\alpha} \pm k_{\beta}\right)} \\
& \times \int_{-\infty}^{\infty} d k \frac{\sqrt{\left|k q_{ \pm}\right|}}{\left(\omega_{1}-v_{k}\right)} \frac{\theta\left[\omega_{2} \pm\left(\omega_{1}-v_{k}\right)\right] e^{i\left(\Delta_{2} \mp \Delta_{1}\right) k}}{4\left(k-k_{\beta}\right)^{2}+\gamma_{\mp}^{2}}
\end{aligned}
$$

For $k_{\beta} \gg \gamma_{\mp}$, the function $1 /\left[4\left(k-k_{\beta}\right)^{2}+\gamma_{\mp}^{2}\right]$ sharply peaks at $k=k_{\beta}$. Approximating the slowly varying factors by their values at $k=k_{\beta}$, the integral in Eq. (16) can be estimated (a)

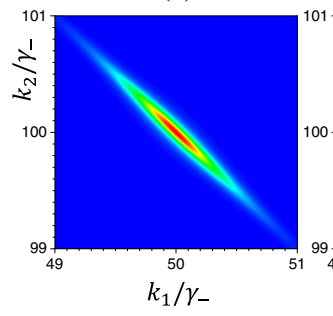

(b)

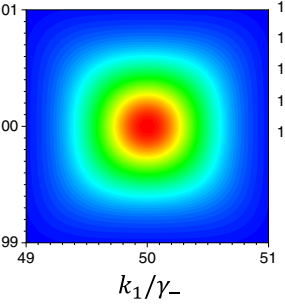

(c)



$\begin{array}{lllllllll}0.1 & 0.2 & 0.3 & 0.4 & 0.5 & 0.6 & 0.7 & 0.8 & 0.9\end{array}$

FIG. 2. Probability to find two photons with wave vectors $k_{1}$ and $k_{2}$ in the state (15) with $k_{\beta}=100 \gamma_{-}, k_{\alpha}=50 \gamma_{-}$, and either $\gamma_{+}=0.1 \gamma_{-}$(a), $\gamma_{+}=\gamma_{-}$(b), or $\gamma_{+}=10 \gamma_{-}$(c). In the plots the probabilities are normalized by their maximum values.

as

$$
\begin{aligned}
A_{ \pm} & \approx \frac{c L_{\mathrm{ph}} N G_{ \pm} e^{i \Delta_{1}\left(k_{\alpha} \pm k_{\beta}\right)} \sqrt{k_{\alpha} k_{\beta}}}{\gamma_{ \pm}^{2}\left(\omega_{1}-c k_{\beta}\right)} \int_{-\infty}^{\infty} \frac{e^{i\left(\Delta_{2} \mp \Delta_{1}\right) k} d k}{4\left(k-k_{\beta}\right)^{2}+\gamma_{\mp}^{2}} \\
& =2 \sqrt{2} \pi c G_{ \pm} e^{i \Delta_{1} k_{\alpha}+i \Delta_{2} k_{\beta}} \frac{\sqrt{k_{\alpha} k_{\beta}}}{\omega_{1}-c k_{\beta}} \sqrt{\frac{\gamma_{\mp}}{\gamma_{ \pm}}} e^{-\left|\Delta_{2} \mp \Delta_{1}\right| \gamma_{\mp} / 2}
\end{aligned}
$$

Equation (17) shows that in the limit $\gamma_{-} \gg \gamma_{+}$and $\mid \Delta_{2}-$ $\Delta_{1} \mid \gamma_{-} \ll 1$, the atom's excitation probability for the ladder scheme is by a factor of $2 \gamma_{-} / \gamma_{+} \gg 1$ greater than that for a separable two-photon state [cf. Eq. (14)]. In contrast, for the Raman $\Lambda$ scheme [lower sign in Eq. (17)], the excitation probability is enhanced if $\gamma_{+} \gg \gamma_{-}$and $\left|\Delta_{2}+\Delta_{1}\right| \gamma_{+} \ll 1$.

The limit $\gamma_{\mp} \gg \gamma_{ \pm}$corresponds to a large frequency correlation or anticorrelation of the state (15). To demonstrate that, in Fig. 2 we plot the probability to find two photons with wave vectors $k_{1}$ and $k_{2}, P\left(k_{1}, k_{2}\right)=\left|\phi\left(k_{1}, k_{2}\right)\right|^{2}$. The figure shows that for $\gamma_{-} \gg \gamma_{+}$the state (15) is frequency anticorrelated [Fig. 2(a)] and it is frequency correlated for $\gamma_{+} \gg \gamma_{-}$[Fig. 2(c)]. For the frequency-correlated (anticorrelated) state the probability $P\left(k_{1}, k_{2}\right)$ peaks along the line $k_{1}=k_{2}+k_{\alpha}-k_{\beta}\left(k_{1}=-k_{2}+k_{\alpha}+k_{\beta}\right)$. If $\gamma_{+} \sim \gamma_{-}$, the correlation disappears. Thus, in order to obtain enhancement of the two-photon excitation in the ladder scheme, the entangled state must be frequency anticorrelated. In contrast, for the Raman scheme, the enhancement occurs if the state is frequency correlated.

The frequency correlation property is independent of $\Delta_{1,2}$, which enters the phase of the state (15). However, according to Eq. (17), to achieve enhancement, one should also satisfy the condition $\left|\Delta_{2} \mp \Delta_{1}\right| \gamma_{\mp} \ll 1$; otherwise, the excitation probability is exponentially suppressed. To gain insight into the latter requirement, we explore the spatial correlation of the entangled state (15). Taking the Fourier transform of Eq. (15), we obtain the following coordinate representation:

$$
\phi \propto e^{i k_{\alpha} x_{1}+i k_{\beta} x_{2}-\gamma_{+}\left|x_{1}+x_{2}+\Delta_{1}+\Delta_{2}\right|-\gamma_{-}\left|x_{1}-x_{2}+\Delta_{1}-\Delta_{2}\right|} .
$$

Equation (18) yields that the probability to find photon 2 at a coordinate $x_{2}$ peaks at $x_{2}=x_{1}+\Delta_{1}-\Delta_{2}$ for $\gamma_{+}<\gamma_{-}$. Thus, if $\Delta_{2}=\Delta_{1}$ and $\gamma_{-} \gg \gamma_{+}$, the state is spatially correlated, and photons arrive simultaneously at the atom's location. For $\gamma_{+}>\gamma_{-}$the probability peaks at 


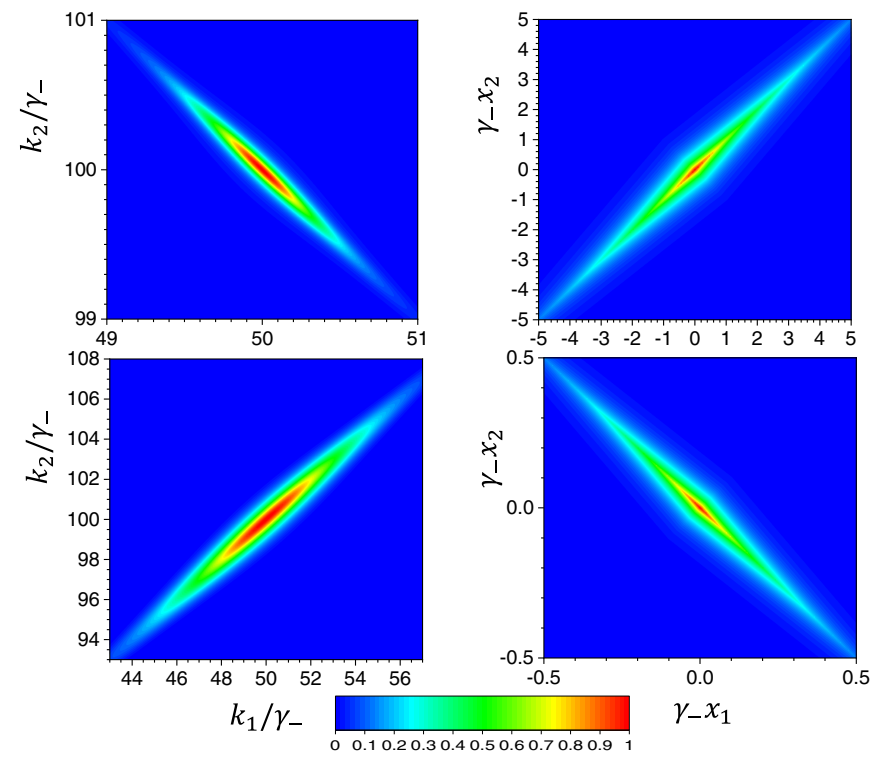

FIG. 3. The left (right) column shows the probability to find two photons in the state (15) with $k_{\beta}=100 \gamma_{-}, k_{\alpha}=50 \gamma_{-}$, and $\Delta_{1,2}=0$ as a function of the photon wave vectors $k_{1}$ and $k_{2}$ (coordinates $x_{1}$ and $x_{2}$ ). For the upper and lower rows, $\gamma_{+}=0.1 \gamma_{-}$and $\gamma_{+}=10 \gamma_{-}$, respectively. In the plots the probabilities are normalized by their maximum values.

$x_{2}=-x_{1}-\Delta_{1}-\Delta_{2}$. Hence, if $\Delta_{2}=-\Delta_{1}$ and $\gamma_{+} \gg \gamma_{-}$, the state is spatially anticorrelated, and two photons always appear symmetrically about the mean time of arrival of the two photons. Since the atom is located at $x=0$, the state is spatially anticorrelated relative to the position of the atom. Please note that we are working in the interaction representation in which the state vector of the free field does not evolve with time and the spatial correlation properties refer to this time-independent state.

In Fig. 3 we plot $\left|\phi\left(k_{1}, k_{2}\right)\right|^{2}$ and $\left|\phi\left(x_{1}, x_{2}\right)\right|^{2}$ for the entangled two-photon state (15) for $\Delta_{1,2}=0$ and either $\gamma_{+}=$ $0.1 \gamma_{-}$(upper row) or $\gamma_{+}=10 \gamma_{-}$(lower row). The figure demonstrates that if the state is frequency anticorrelated (upper row), it is spatially correlated, and vice versa.

A frequency-anticorrelated state can be produced in a cascade decay of an atomic three-level system with transition frequencies $c k_{\alpha}$ and $c k_{\beta}$ [32]. For such a state,

$$
\phi\left(k_{1}, k_{2}\right)=\frac{2 \sqrt{\gamma_{\alpha} \gamma_{\beta}} e^{-i x_{0}\left(k_{1}+k_{2}\right)}}{L_{\mathrm{ph}}\left(k_{1}+k_{2}-k_{\alpha}-k_{\beta}+i \gamma_{\alpha}\right)\left(k_{2}-k_{\beta}+i \gamma_{\beta}\right)},
$$

where $\gamma_{\alpha}$ and $\gamma_{\beta}$ are the half-widths of the upper and lower transition in the decaying atom, respectively, and $x_{0}$ is the location of the source. In Fig. 4 we plot the probability to find two photons with wave vectors $k_{1}$ and $k_{2}$ for the state (19). The figure shows that for $\gamma_{\beta} \gg \gamma_{\alpha}$ the state (19) is frequency anticorrelated, while for $\gamma_{\beta} \lesssim \gamma_{\alpha}$ the correlation disappears. For $\gamma_{\beta} \gg \gamma_{\alpha}$ the excitation probability of the ladder system of Fig. 1(a) by the pulse (19) is enhanced by a factor of $\gamma_{\beta} / \gamma_{\alpha}$ as compared with that for separable states.

The enhancement occurs because for $\gamma_{\beta} \gg \gamma_{\alpha}$ the state (19) is also spatially correlated, and pairs of the entangled photons are emitted at very nearly the same time. This (a)

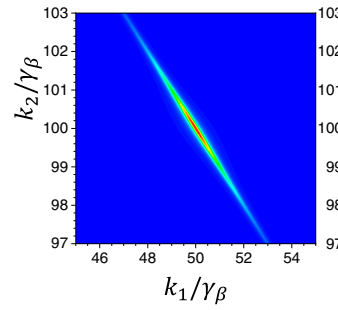

(b)

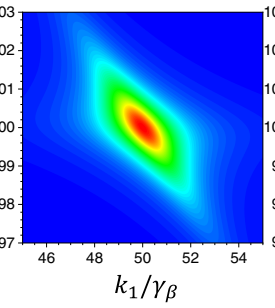

(c)

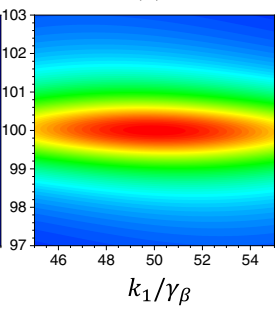

FIG. 4. Probability to find two photons with wave vectors $k_{1}$ and $k_{2}$ in the state (19) with $k_{\beta}=100 \gamma_{\beta}, k_{\alpha}=50 \gamma_{\beta}$, and either $\gamma_{\alpha}=$ $0.1 \gamma_{\beta}$ (a), $\gamma_{\alpha}=\gamma_{\beta}$ (b), or $\gamma_{\alpha}=10 \gamma_{\beta}$ (c). In the plots the probabilities are normalized by their maximum values.

allows both photons to simultaneously interact with a given atom, which enhances the rate of two-photon absorption $[12,21,30,33]$. The atom's excitation is accomplished in a single step: One photon of the pair promotes the atom to the virtual intermediate state, while its counterpart immediately (in a time less than the virtual-state lifetime) completes the two-photon transition [13].

Our results can be straightforwardly generalized to the case of a many-photon entangled pulse described by the field state vector

$$
|i\rangle=\sum_{\mathbf{k}_{1}, \mathbf{k}_{2}} \phi\left(\mathbf{k}_{1}, \mathbf{k}_{2}\right)\left|n_{\mathbf{k}_{1}} m_{\mathbf{k}_{2}}\right\rangle,
$$

where $\phi\left(\mathbf{k}_{1}, \mathbf{k}_{2}\right)$ is a probability amplitude to find the field in the state with $n_{\mathbf{k}_{1}}$ photons in the mode $\mathbf{k}_{1}$ and $m_{\mathbf{k}_{2}}$ photons in the mode $\mathbf{k}_{2}$. We find that in this case the excitation probability amplitude is described by Eqs. (14) and (17) multiplied by $\sqrt{n_{k_{\alpha}} m_{k_{\beta}}}$ for the ladder scheme and $\sqrt{\left(n_{k_{\alpha}}+1\right) m_{k_{\beta}} / 2}$ for the Raman $\Lambda$ scheme. That is, the conclusion about enhancement of the excitation probability by a factor of $2 \gamma_{\mp} / \gamma_{ \pm}$ remains true for the more general field state (20). Thus use of frequency-correlated (anticorrelated) entangled states of light is advantageous not only for small pulse intensity, but also for many-photon pulses. This makes it more appealing for practical applications.

In summary, we consider stimulated Raman excitation and two-photon absorption by a three-level system interacting with a two-photon pulse. We obtain analytically an upper limit on the probability of excitation produced by separable twophoton states and an expression for the excitation probability by an entangled pulse.

We find that stimulated Raman process can be enhanced by orders of magnitude if photons in the pulse are simultaneously frequency correlated and spatially anticorrelated. That is, a correlated photon pair must have an inherent time delay between its constituent photons; yet the stimulated Raman process is enhanced. This counterintuitive feature is a consequence of the quantum-mechanical uncertainty principle, which in the operator form reads $\Delta A \Delta B \geqslant \frac{1}{2}|\langle[\hat{A}, \hat{B}]\rangle|$, where $\Delta A$ and $\Delta B$ are standard deviations of $\hat{A}$ and $\hat{B}$. Taking $\hat{A}=$ $\hat{x}_{1}-\hat{x}_{2}$ and $\hat{B}=\hat{k}_{1}-\hat{k}_{2}$, where $\hat{k}=i \partial / \partial x$ is the wave-vector operator, we obtain $[\hat{A}, \hat{B}]=-2 i$, and the uncertainty principle yields $\Delta\left(x_{1}-x_{2}\right) \geqslant 1 / \Delta\left(k_{1}-k_{2}\right)$. That is, if photons 
are frequency correlated [ $\Delta\left(k_{1}-k_{2}\right)$ is small], they cannot be spatially correlated.

This is opposite to two-photon absorption, for which the enhancement occurs if photons in the pulse are frequency anticorrelated and spatially correlated, that is, photons in the pair simultaneously interact with the atom. Since operators $\hat{k}_{1}+\hat{k}_{2}$ and $\hat{x}_{1}-\hat{x}_{2}$ commute, the uncertainty principle allows photons to be simultaneously frequency anticorrelated and time (spatially) correlated. We show that it is important to have frequency and spatial correlation (or anticorrelation) simultaneously in order to achieve enhancement of the two-photon processes for the ladder and the Raman schemes.

In the present analysis we consider two-photon pulses and study the effect of frequency and space correlation. If we allow pulses to have an arbitrary number of photons, the problem becomes richer. For example, if the average number of photons in the pulse is much smaller than 1 , then entanglement can enhance two-photon absorption even if photons in the pulse are not frequency correlated, but photon numbers are correlated (see Appendix for details). However, use of frequency-correlated (anticorrelated) entangled states of light is also advantageous for many-photon pulses.

Our findings can be useful for imaging and spectroscopy of biological samples which demand low illumination intensity $[10,11]$, and the possibility of measurement of ground-state hyperfine transitions [34-36]. Our result [see Eq. (17)] also shows that frequency entanglement can be used for suppression of unwanted two-photon processes. For example, if photons in the pulse are frequency correlated $\left(\gamma_{-} \ll \gamma_{+}\right)$, then the two-photon excitation probability is suppressed by a factor of $\gamma_{-} / \gamma_{+} \ll 1$, while the Raman process is not. Similarly, if photons are frequency anticorrelated, then stimulated Raman excitation is suppressed. Thus entanglement can be used to control the rate of atomic excitation and select desired excitation pathways. One should mention that Raman or two-photon absorption pathways can be also selectively eliminated by using peculiar entangled states generated by sending a twophoton pulse through an interferometer [37-39]. Schemes in which only one photon from the pair is used to excite the sample, while the other photon serves as a reference, have also been investigated $[38,40]$. In addition, it has been shown experimentally that efficiency of coherent Raman processes depends on the classical correlation between the driving fields [41,42].

Owing to the recent developments in nonlinear crystal optics, a high degree of frequency correlation or anticorrelation can be achieved by use of special phase-matching conditions in a spontaneous parametric frequency downconversion process. For example, the photons generated with a continuous-wave pump are frequency anticorrelated. On the other hand, frequency-correlated photons can be obtained in a special nonlinear crystal in which the group velocity of the pump beam happens to match the average group velocity of the downconverted photons $[43,44]$. In fact, generation of the correlated photons has been demonstrated [45-48], where the frequency correlation and anticorrelation can be controlled by artificially designed material with quasi-phase-matching, or by other means [49-51].

\section{ACKNOWLEDGMENTS}

M.O.S. wishes to thank Prof. J. T. Shen for stimulating his interest in this problem. This work was supported by the National Science Foundation (Award No. PHY-2013771), the Air Force Office of Scientific Research (Award No. FA955020-1-0366 DEF), the Office of Naval Research (Awards No. N00014-20-1-2184 and No. N00014-16-1-2578), the Robert A. Welch Foundation (Awards No. A-1261, No. A-1547, No. A-1801, and No. A-1943), the Russian Foundation for Basic Research (Projects No. 18-29-20031 and No. 19-0200473), and the Russian Science Foundation (Project No. 20-12-00088: Broadband Nonlinear Optics). A.C. acknowledges support from the Alexander von Humboldt Foundation in the framework of a Feodor Lynen Research Fellowship.

\section{APPENDIX: ATOM EXCITATION BY ENTANGLED LIGHT WITH NO FREQUENCY CORRELATION}

Here, we show that photon entanglement can enhance twophoton processes even if there is no frequency correlation between photons. We consider the ladder scheme of Fig. 1(a) and the Raman scheme of Fig. 1(b) and assume that the field is a superposition of two modes described by mode functions $\Phi_{1}(t, \mathbf{r})$ and $\Phi_{2}(t, \mathbf{r})$ which have carrier frequencies close to $\omega_{1}$ and $\omega_{2}$, respectively. The mode functions $\Phi_{1}$ and $\Phi_{2}$ are properly normalized.

In the rotating wave approximation the interaction Hamiltonian between the three-level atom located at $\mathbf{r}=0$ and the two-mode field for the ladder scheme reads

$$
\begin{aligned}
\hat{V}(t)= & -\wp_{b c} g_{1} \Phi_{1}^{*}(t, 0) e^{i \omega_{1} t} \hat{a}_{1}|b\rangle\langle c| \\
& -\wp_{a b} g_{2} \Phi_{2}^{*}(t, 0) e^{i \omega_{2} t} \hat{a}_{2}|a\rangle\langle b|+\text { H.c. },
\end{aligned}
$$

where $\hat{a}_{1,2}$ and $\hat{a}_{1,2}^{\dagger}$ are annihilation and creation operators for the photon in modes 1 and $2, \wp_{b c}$ and $\wp_{a b}$ are dipole moment matrix elements between the states $|b\rangle$ and $|c\rangle$, and $|a\rangle$ and $|b\rangle$, respectively, and $g_{1}$ and $g_{2}$ are the coupling constants between the atom and modes 1 and 2 . For the $\Lambda$ scheme, the second term in Eq. (A1) should be replaced with

$$
-\wp_{a b} g_{2} \Phi_{2}(t, 0) e^{-i \omega_{2} t} \hat{a}_{2}^{\dagger}|a\rangle\langle b| .
$$

Substituting Eq. (A1) into Eq. (2) yields

$$
\begin{aligned}
A_{+}= & -\frac{1}{\hbar^{2}} \wp_{a b} \wp_{b c} g_{1} g_{2} M_{+} \\
& \times \int_{-\infty}^{\infty} \Phi_{2}^{*}\left(t^{\prime}, 0\right) e^{i \omega_{2} t^{\prime}} d t^{\prime} \int_{-\infty}^{t^{\prime}} \Phi_{1}^{*}\left(t^{\prime \prime}, 0\right) e^{i \omega_{1} t^{\prime \prime}} d t^{\prime \prime}
\end{aligned}
$$

for the ladder scheme and

$$
\begin{aligned}
A_{-}= & -\frac{1}{\hbar^{2}} \wp_{a b \wp \rho c} g_{1} g_{2} M_{-} \\
& \times \int_{-\infty}^{\infty} \Phi_{2}\left(t^{\prime}, 0\right) e^{-i \omega_{2} t^{\prime}} d t^{\prime} \int_{-\infty}^{t^{\prime}} \Phi_{1}^{*}\left(t^{\prime \prime}, 0\right) e^{i \omega_{1} t^{\prime \prime}} d t^{\prime \prime}
\end{aligned}
$$

for the Raman scheme, where

$$
M_{+}=\sum_{f}\left\langle f\left|\hat{a}_{2} \hat{a}_{1}\right| i\right\rangle, \quad M_{-}=\sum_{f}\left\langle f\left|\hat{a}_{2}^{\dagger} \hat{a}_{1}\right| i\right\rangle .
$$


We assume that for the Raman scheme the Stokes photon is emitted in mode 2.

If we are interested in the probability amplitude that the atom goes from the ground state $|c\rangle$ to the state $|a\rangle$ without specifying the final state of the field, then the sum in Eq. (A4) should be taken over a complete set of final states of the field $|f\rangle$. As a result, we obtain

$$
\left|M_{+}\right|^{2}=\left\langle i\left|\hat{a}_{2}^{\dagger} \hat{a}_{2} \hat{a}_{1}^{\dagger} \hat{a}_{1}\right| i\right\rangle, \quad\left|M_{-}\right|^{2}=\left\langle i\left|\hat{a}_{2} \hat{a}_{2}^{\dagger} \hat{a}_{1}^{\dagger} \hat{a}_{1}\right| i\right\rangle .
$$

Equations (A2) and (A3) show that the transition probability is proportional to $P_{ \pm} \propto\left|M_{ \pm}\right|^{2}$ and dependence on the pulse shapes and their overlap is factored out.

If the initial state of the field is separable, that is, $|i\rangle$ can be written as a product of the field state in modes 1 and 2, $|i\rangle=\left|i_{1}\right\rangle\left|i_{2}\right\rangle$, then Eq. (A5) yields

$$
\left|M_{+}\right|^{2}=\bar{n}_{1} \bar{n}_{2}, \quad\left|M_{-}\right|^{2}=\bar{n}_{1}\left(\bar{n}_{2}+1\right),
$$

where $\bar{n}_{1}$ and $\bar{n}_{2}$ are the average numbers of photons in modes 1 and 2 , respectively. That is, for a separable state, the excitation probability goes as

$$
P_{+} \propto \bar{n}_{1} \bar{n}_{2}, \quad P_{-} \propto \bar{n}_{1}\left(\bar{n}_{2}+1\right) .
$$

If the initial state of the field is entangled, for example, it is a two-mode squeezed state

$$
|i\rangle=e^{\alpha^{*} \hat{a}_{1}^{\dagger} \hat{a}_{2}^{\dagger}+\alpha \hat{a}_{1} \hat{a}_{2}}\left|0_{1} 0_{2}\right\rangle,
$$

where $\alpha$ is a parameter, then

$$
\left|M_{+}\right|^{2}=\bar{n}(1+2 \bar{n}), \quad\left|M_{-}\right|^{2}=2 \bar{n}(1+\bar{n}) .
$$

That is, the excitation probability goes as the average photon number in the mode, $P_{ \pm} \propto \bar{n}$, for $\bar{n} \ll 1$, and as $\bar{n}^{2}$ for $\bar{n} \gg 1$.

The two-mode squeezed state can be generated in a nonlinear crystal by a parametric down-conversion process in an optical cavity [52] or utilizing a four-wave mixing process in an atomic vapor [27]. For this state, the photon numbers in modes are correlated. Namely, the photon numbers in each mode $n_{1}$ and $n_{2}$ fluctuate obeying thermal distribution, but the difference $n_{1}-n_{2}$ does not fluctuate. That is, if there are $n$ photons in mode 1 , then with unit probability there are $n$ photons in mode 2 .

Equation (A7) shows that entangled light (with photon number correlation) can yield substantially higher probability of the two-photon absorption for low illumination intensity compared with a separable state [see Eq. (A6)]. In the present example there is no frequency correlation between photons in the two modes. Frequency-correlated photons can yield an additional enhancement, as discussed in the main text.

One should mention that the Minkowski vacuum is a squeezed state if the field is described using Rindler modes [53]. In $1+1$ dimensions, the Rindler mode functions read

$$
\begin{aligned}
\phi_{1 v} & =\sqrt{\frac{a}{v c}}(\mp z-c t)^{i \frac{v c}{a}} \theta(\mp z-c t), \\
\phi_{2 v} & =\sqrt{\frac{a}{v c}}(c t \pm z)^{-i \frac{v c}{a}} \theta(c t \pm z) .
\end{aligned}
$$

Here, $v$ is the photon frequency in the Rindler space, and $a$ is a parameter which has the dimension of the acceleration. For $v>0$ the modes (A8) and (A9) have a positive norm. The upper and lower signs in Eqs. (A8) and (A9) correspond to the

\section{Minkowski space}

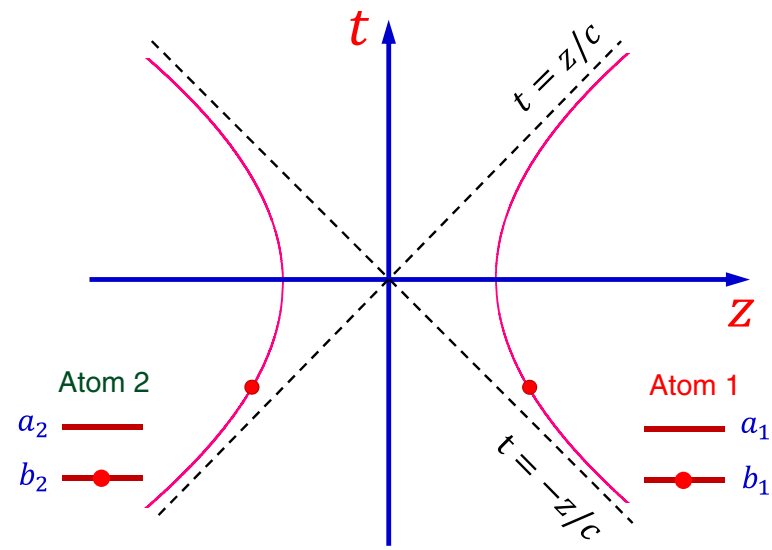

FIG. 5. Ground-state atoms 1 and 2 are uniformly accelerated in the right and left Rindler wedges, respectively, and become excited simultaneously.

left- and right-propagating photons, respectively. The mode functions (A8) and (A9) are nonzero in half of the $t-z$ plane and form a complete basis set.

Introducing operators of the Rindler photons $\hat{b}_{v}$, one can obtain for the Minkowski vacuum $\left|0_{M}\right\rangle$ [53]

$$
\left\langle 0_{M}\left|\hat{b}_{1 v}^{\dagger} \hat{b}_{1 v}\right| 0_{M}\right\rangle=\left\langle 0_{M}\left|\hat{b}_{2 v}^{\dagger} \hat{b}_{2 v}\right| 0_{M}\right\rangle=\frac{1}{e^{2 \pi c v / a}-1} .
$$

That is, the Minkowski vacuum is filled with Rindler photons; however, their number for realistic parameters is exponentially small. In the Minkowski vacuum the number of Rindler photons in the modes $\phi_{1 v}$ and $\phi_{2 v}$ is correlated. One can obtain the following representation of the Minkowski vacuum in terms of Rindler states [53]:

$$
\begin{aligned}
\left|0_{M}\right\rangle= & \prod_{v>0}\left(1-e^{-2 \pi c v / a}\right) e^{\exp \left(-\frac{\pi c v}{a}\right)\left(\hat{b}_{R 1 v}^{\dagger} \hat{b}_{R 2 v}^{\dagger}+\hat{b}_{L 1 v}^{\dagger} \hat{b}_{L 2 v}^{\dagger}\right)}\left|0_{R}\right\rangle \\
= & \prod_{v>0}\left(1-e^{-2 \pi c v / a}\right) \\
& \sum_{\substack{n_{R 1 v}=n_{R 2 v}=0 \\
n_{L 1 v}=n_{L 2 v}=0}}^{\infty} e^{-\pi\left(n_{R 1 v}+n_{L 1 v}\right) c v / a}\left|n_{R 1 v}, n_{R 2 v}\right\rangle \\
& \times\left|n_{L 1 v}, n_{L 2 v}\right\rangle,
\end{aligned}
$$

where $\left|n_{R 1 v}, n_{R 2 v}\right\rangle\left(\left|n_{L 1 v}, n_{L 2 v}\right\rangle\right)$ are states with $n_{R 1 v}$ and $n_{R 2 v}$ $\left(n_{L 1 v}\right.$ and $\left.n_{L 2 v}\right)$ Rindler photons in the right-propagating (leftpropagating) modes $\phi_{1 v}$ and $\phi_{2 v}$. That is, if there are $n$ Rindler photons in the right-propagating (left-propagating) mode $\phi_{1 v}$, then with unit probability there are $n$ Rindler photons in the right-propagating (left-propagating) mode $\phi_{2 v}$, and vice versa.

From the perspective of an atom uniformly accelerated with acceleration $a$, the frequencies of Rindler photons are positive [54]. Such a ground-state atom can absorb Rindler photons and become excited, which is known as the FullingDavies-Unruh effect. If two ground-state atoms are uniformly 
accelerated in opposite directions (see Fig. 5), they can become excited by absorbing Rindler photons from the modes $\phi_{1 v}$ and $\phi_{2 v}$, respectively.

Due to vacuum correlations, if atom 2 becomes excited by absorbing the right-propagating Rindler photon $\phi_{2 \omega}$ of frequency $\omega$, then with unit probability there is a nonzero number of the right-propagating Rindler photons in the mode $\phi_{1 \omega}$. They can excite atom 1 . That is, atom 1 becomes ex- cited with a much higher probability provided that atom 2 detected a photon. Because of the photon number correlation, the joint excitation probability of two atoms is proportional to the average number of Rindler photons, rather than the average number squared. This is analogous to the two-photon absorption of a stationary atom illuminated by squeezed light, for which, in the limit $\bar{n} \ll 1$, the excitation probability goes as $P \propto \bar{n}$.
[1] J. D. Franson, B. C. Jacobs, and T. B. Pittman, Quantum computing using single photons and the Zeno effect, Phys. Rev. A 70, 062302 (2004).

[2] B. C. Jacobs and J. D. Franson, All-optical switching using the quantum Zeno effect and two-photon absorption, Phys. Rev. A 79, 063830 (2009).

[3] W. Denk, J. H. Strickler, and W. W. Webb, Two-photon laser scanning fluorescence microscopy, Science 248, 73 (1990).

[4] P. Kok, S. L. Braunstein, and J. P. Dowling, Quantum lithography, entanglement and Heisenberg-limited parameter estimation, J. Opt. B: Quantum Semiclassical Opt. 6, S811 (2004).

[5] K. Gazeli, G. Lombardi, X. Aubert, C. Y. Duluard, S. Prasanna, and K. Hassouni, Progresses on the use of two-photon absorption laser induced fluorescence diagnostics for measuring absolute atomic densities in plasmas and flames, Plasma 4, 145 (2021).

[6] J. Waskoenig, K. Niemi, N. Knake, L. M. Graham, S. Reuter, V. Schulz-von der Gathen, and T. Gans, Atomic oxygen formation in a radio-frequency driven micro-atmospheric pressure plasma jet, Plasma Sources Sci. Technol. 19, 045018 (2010).

[7] N. Knake, K. Niemi, S. Reuter, V. Schulz-von der Gathen, and J. Winter, Absolute atomic oxygen density profiles in the discharge core of a microscale atmospheric pressure plasma jet, Appl. Phys. Lett. 93, 131503 (2008).

[8] E. Wagenaars, T. Gans, D. O'Connell, and K. Niemi, Twophoton absorption laser-induced fluorescence measurements of atomic nitrogen in a radio-frequency atmospheric-pressure plasma jet, Plasma Sources Sci. Technol. 21, 042002 (2012).

[9] J. B. Schmidt, S. Roy, W. D. Kulatilaka, I. Shkurenkov, I. V. Adamovich, W. R. Lempert, and J. R. Gord, Femtosecond, two-photon-absorption, laser-induced-fluorescence imaging of atomic hydrogen and oxygen in non-equilibrium plasmas, J. Phys. D: Appl. Phys. 50, 015204 (2017).

[10] O. Varnavski and Th. Goodson, Two-photon fluorescence microscopy at extremely low excitation intensity: The power of quantum correlations, J. Am. Chem. Soc. 142, 12966 (2020).

[11] J. P. Villabona-Monsalve, O. Varnavski, B. A. Palfey, and Th. Goodson, Two-photon excitation of flavins and flavoproteins with classical and quantum light, J. Am. Chem. Soc. 140, 14562 (2018).

[12] J. Gea-Banacloche, Two-Photon Absorption of Nonclassical Light, Phys. Rev. Lett. 62, 1603 (1989).

[13] J. Javanainen and P. L. Gould, Linear intensity dependence of a two-photon transition rate, Phys. Rev. A 41, 5088 (1990).

[14] N. P. Georgiades, E. S. Polzik, K. Edamatsu, H. J. Kimble, and A. S. Parkins, Nonclassical Excitation for Atoms in a Squeezed Vacuum, Phys. Rev. Lett. 75, 3426 (1995).
[15] H. Oka, Real-time analysis of two-photon excitation by correlated photons: Pulse-width dependence of excitation efficiency, Phys. Rev. A 81, 063819 (2010).

[16] H. Oka, Selective two-photon excitation of a vibronic state by correlated photons, J. Chem. Phys. 134, 124313 (2011).

[17] Y. Zhou, Z. Chen, L. V. Wang, and J.-T. Shen, Efficient two-photon excitation by photonic dimers, Opt. Lett. 44, 475 (2019).

[18] B. Gu and S. Mukamel, Manipulating two-photon-absorption of cavity polaritons by entangled light, J. Phys. Chem. Lett. 11, 8177 (2020).

[19] H. Oka, Efficient two-step up-conversion by quantumcorrelated photon pairs, Opt. Express 18, 25839 (2010).

[20] M. Nakatani, R. Shimizu, and K. Koshino, Up-conversion dynamics for temporally entangled two-photon pulses, Phys. Rev. A 83, 013824 (2011).

[21] B. Dayan, A. Pe'er, A. A. Friesem, and Y. Silberberg, Two Photon Absorption and Coherent Control with Broadband Down-Converted Light, Phys. Rev. Lett. 93, 023005 (2004).

[22] D. Lee and T. Goodson, Entangled photon absorption in an organic porphyrin dendrimer, J. Phys. Chem. B 110, 25582 (2006).

[23] A. Muthukrishnan, G. S. Agarwal, and M. O. Scully, Inducing Disallowed Two-Atom Transitions with Temporally Entangled Photons, Phys. Rev. Lett. 93, 093002 (2004).

[24] W. Ren, W. Liu, C. Song, H. Li, Q. Guo, Z. Wang, D. Zheng, G. S. Agarwal, M. O. Scully, S.-Y. Zhu, H. Wang, and D.-W. Wang, Simultaneous Excitation of Two Noninteracting Atoms with Time-Frequency Correlated Photon Pairs in a Superconducting Circuit, Phys. Rev. Lett. 125, 133601 (2020).

[25] H.-B. Fei, B. M. Jost, S. Popescu, B. E. A. Saleh, and M. C. Teich, Entanglement-Induced Two-Photon Transparency, Phys. Rev. Lett. 78, 1679 (1997).

[26] J. You, Z. Liao, and M. S. Zubairy, Steady-state population inversion of multiple $\Xi$-type atoms by the squeezed vacuum in a waveguide, Phys. Rev. A 100, 013843 (2019).

[27] T. Li, F. Li, C. Altuzarra, A. Classen, and G. S. Agarwal, Squeezed light induced two-photon absorption fluorescence of fluorescein biomarkers, Appl. Phys. Lett. 116, 254001 (2020).

[28] P. G. Kwiat, K. Mattle, H. Weinfurter, A. Zeilinger, A. V. Sergienko, and Y. Shih, New High-Intensity Source of Polarization-Entangled Photon Pairs, Phys. Rev. Lett. 75, 4337 (1995).

[29] J. Janszky and Y. Yushin, Many-photon processes with the participation of squeezed light, Phys. Rev. A 36, 1288 (1987).

[30] B. Dayan, Theory of two-photon interactions with broadband down-converted light and entangled photons, Phys. Rev. A 76, 043813 (2007). 
[31] O. Varnavski, B. Pinsky, and T. Goodson, Entangled photon excited fluorescence in organic materials: an ultrafast coincidence detector, J. Phys. Chem. Lett. 8, 388 (2017).

[32] M. O. Scully and S. Zubairy, Quantum Optics (Cambridge University Press, Cambridge, 1997).

[33] H. You, S. M. Hendrickson, and J. D. Franson, Enhanced two-photon absorption using entangled states and small mode volumes, Phys. Rev. A 80, 043823 (2009).

[34] E. Gomez, S. Aubin, G. D. Sprouse, L. A. Orozco, and D. P. DeMille, Measurement method for the nuclear anapole moment of laser-trapped alkali-metal atoms, Phys. Rev. A 75, 033418 (2007).

[35] D. Sheng, L. A. Orozco, and E. Gomez, Preliminary studies for anapole moment measurements in rubidium and francium, J. Phys. B: At., Mol. Opt. Phys. 43, 074004 (2010).

[36] J. Choi and D. S. Elliott, Measurement scheme and analysis for weak ground-state-hyperfine-transition moments through two-pathway coherent control, Phys. Rev. A 93, 023432 (2016).

[37] O. Roslyak, C. A. Marx, and S. Mukamel, Nonlinear spectroscopy with entangled photons: Manipulating quantum pathways of matter, Phys. Rev. A 79, 033832 (2009).

[38] K. E. Dorfman, F. Schlawin, and S. Mukamel, Nonlinear optical signals and spectroscopy with quantum light, Rev. Mod. Phys. 88, 045008 (2016).

[39] P. Munkhbaatar and K. Myung-Whun, Selection of stimulated Raman scattering signal by entangled photons, Opt. Commun. 383, 581 (2017).

[40] K. E. Dorfman, F. Schlawin, and S. Mukamel, Stimulated Raman spectroscopy with entangled light: Enhanced resolution and pathway selection, J. Phys. Chem. Lett. 5, 2843 (2014).

[41] Z. W. Li, C. Radzewicz, and M. G. Raymert, Phase cross correlation in the coherent Raman process, Opt. Lett. 13, 491 (1988).

[42] Z. W. Li, C. Radzewicz, and M. G. Raymert, Cancellation of laser phase fluctuations in Stokes and anti-Stokes generation, J. Opt. Soc. Am. B 5, 2340 (1988).
[43] R. Erdmann, D. Branning, W. Grice, and I. A. Walmsley, Restoring dispersion cancellation for entangled photons produced by ultrashort pulses, Phys. Rev. A 62, 053810 (2000).

[44] V. Giovannetti, L. Maccone, J. H. Shapiro, and F. N. C. Wong, Generating Entangled Two-Photon States with Coincident Frequencies, Phys. Rev. Lett. 88, 183602 (2002).

[45] J. P. Torres, F. Macià, S. Carrasco, and L. Torner, Engineering the frequency correlations of entangled two-photon states by achromatic phase matching, Opt. Lett. 30, 314 (2005).

[46] M. Hendrych, M. Micuda, and J. P. Torres, Tunable control of the frequency correlations of entangled photons, Opt. Lett. 32, 2339 (2007).

[47] R. Shimizu and K. Edamatsu, High-flux and broadband biphoton sources with controlled frequency entanglement, Opt. Express 17, 16385 (2009).

[48] B. J. Smith, P. Mahou, O. Cohen, J. S. Lundeen, and I. A. Walmsley, Photon pair generation in birefringent optical fibers, Opt. Express 17, 23589 (2009).

[49] A. Valencia, A. Ceré, X. Shi, G. Molina-Terriza, and J. P. Torres, Shaping the Waveform of Entangled Photons, Phys. Rev. Lett. 99, 243601 (2007).

[50] X. Caillet, V. Berger, G. Leo, and S. Ducci, A semiconductor source of counterpropagating twin photons: a versatile device allowing the control of the two-photon state, J. Mod. Opt. 56 232 (2009).

[51] F. Hou, X. Xiang, R. Quan, M. Wang, Y. Zhai, S. Wang, T. Liu, S. Zhang, and R. Dong, An efficient source of frequency anticorrelated entanglement at telecom wavelength, Appl. Phys. B: Lasers Opt. 122, 128 (2016).

[52] L.-A. Wu, H. J. Kimble, J. L. Hall, and H. Wu, Generation of Squeezed States by Parametric Down Conversion, Phys. Rev. Lett. 57, 2520 (1986).

[53] A. A. Svidzinsky, A. Azizi, J. S. Ben-Benjamin, M. O. Scully, and W. Unruh, Causality in quantum optics and entanglement of Minkowski vacuum, Phys. Rev. Research 3, 013202 (2021).

[54] A. A. Svidzinsky, A. Azizi, J. S. Ben-Benjamin, M. O. Scully, and W. Unruh, Unruh and Cherenkov Radiation from a Negative Frequency Perspective, Phys. Rev. Lett. 126, 063603 (2021). 